\title{
Effect of oxytocin infusion versus tranexamic acid on reducing blood loss during hysteroscopic myomectomy: a randomized controlled trial
}

\author{
Sabry E. Mohamed, Amr M. El Helaly, Mohamed H. Salama*
}

Department of Obstetrics and Gynecology, Faculty of Medicine, Ain Shams University, Cairo, Egypt

Received: 28 May 2019

Accepted: 03 June 2019

\section{*Correspondence:}

Dr. Mohamed H. Salama,

E-mail: mhs_vagus2009@yahoo.com

Copyright: () the author(s), publisher and licensee Medip Academy. This is an open-access article distributed under the terms of the Creative Commons Attribution Non-Commercial License, which permits unrestricted non-commercial use, distribution, and reproduction in any medium, provided the original work is properly cited.

\begin{abstract}
Background: Women undergoing hysteroscopic myomectomy are prone to significant blood loss and hematological disturbances. Oxytocin is a uterotonic drug, used mainly in obstetric practice. Tranexamic acid is a haemostatic drug that has been used to reduce blood loss after trauma and in many surgeries. The aim of our study was to compare the safety and efficacy of oxytocin versus tranexamic acid in reducing perioperative blood loss during hysteroscopic myomectomy.

Methods: This study included 60 patients scheduled for hysteroscopic myomectomy allocated into 2 groups: group A: received $10 \mathrm{mg} / \mathrm{kg}$ of tranexamic acid slowly intravenous after induction of anesthesia, while group B: received infusion of $10 \mathrm{IU}$ of oxytocin at a rate of $400 \mathrm{mIU} / \mathrm{min}$ throughout the procedure. Preoperative and postoperative hemoglobin and hematocrit levels, need for blood transfusion, duration of operation, complications and medications adverse effects were recorded.

Results: The 2 groups were comparable in terms of the mean difference between preoperative and postoperative hemoglobin a hematocrit levels, operative time, operative complication and medications' adverse effects. No patients needed blood component transfusion in either group.

Conclusions: It can be concluded that tranexamic acid and oxytocin are equally effective in reducing blood loss and transfusion requirements during hysteroscopic myomectomy with comparable good safety profiles. Both of them can be used according to availability and surgeon preferences.
\end{abstract}

Keywords: Hysteroscopic myomectomy, Myomectomy, Oxytocin, Reducing blood loss, Tranexamic acid

\section{INTRODUCTION}

Uterine fibroids are benign, smooth muscle tumours of the uterus that are affected by the levels of estrogen and progesterone. ${ }^{1}$ Fibroids are the most common female pelvic tumors, and these can be sonographically identified by ultrasound in up to $70-80 \%$ of women before menopause. ${ }^{2}$

Most fibroids are asymptomatic; however, 20-50 percent may be symptomatic causing abnormal uterine bleeding, congestive dysmenorrhea, pressure manifestations, infertility or recurrent pregnancy loss, especially when these are submucou. ${ }^{3}$ The standard treatment for symptomatising myomas is hysterectomy for women with completed families, and myomectomy for those who wish to preserve their fertility. ${ }^{4}$ Myomectomy can be performed by laparotomy, laparoscopy or hysteroscopy according to the size, site and number of the myomas. ${ }^{5}$

Hysteroscopic myomectomy is currently considered the "gold standard" minimally invasive approach for the 
treatment of symptomatic submucous myomas. ${ }^{6}$ Patients undergoing hysteroscopic myomectomy are liable to significant blood loss, and hemodynamic and hematological disturbances. Many interventions were introduced to reduce the risk of bleeding during myomectomy. These include the use of utero-tonics such as oxytocin, or the use of anti-fibrinolytics such as tranexamic acid. ${ }^{7}$

Oxytocin is a utero-tonic agent that causes myometrial contraction. The potential advantage of oxytocin infusion during hysteroscopic myomectomy is that it can maintains uterine contractility throughout the procedure, and thus, reduce blood loss. ${ }^{8}$

Tranexamic acid is a synthetic derivative of the lysine amino acid, which can exert an antifibrinolytic effect via the reversible blockage of the lysine-binding sites on plasminogen. This inhibits its proteolytic action on fibrin, thus, prevents further fibrinolysis and maintains the clots. Hence, it decreases the blood loss during surgery. ${ }^{9}$ Intravenous administration of tranexamic acid has been used for decades to reduce bleeding during after surgical procedures, especially ones like coronary artery graft bypass, liver transplantation, hip or knee arthroplasty. ${ }^{10}$

Excessive bleeding during hysteroscopic myomectomy remains a major challenge for the endoscopic gynaecological surgeons. Although many interventions have been described to reduce the intraoperative blood loss, there is a need for a well-designed randomised controlled trials to identify the most efficient interventions, with reasonable safety profiles, to help the perform a safe and curative surgery. ${ }^{7}$

\section{METHODS}

This study was designed to compare the safety and efficacy of oxytocin and tranexamic acid in reducing the perioperative blood loss and transfusion requirements during hysteroscopic myomectomy.

This randomized controlled trial was conducted at the Early Cancer Detection Unit (ECDU) of the Ain Shams University Maternity Hospital, from September 2017 through August 2018. It was approved by Ethical Committee of the Department of Obstetrics and Gynaecology, Faculty of Medicine, Ain Shams University.

This study included patients aged 18-45 years with symptomatic submucous myomas. Patients included were diagnosed by transvaginal ultrasonography to have 1 or 2 submucous uterine myomas with a diameter between 10 and $40 \mathrm{~mm}$ that were FIGO Grade 0 or 1 .

Patients with a hemoglobin level less than $100 \mathrm{gm} / \mathrm{L}$ were excluded from the study, as well as those with chronic medical diseases, bleeding disorders, hypercoagulopathy, history of thromboembolism, known allergy to tranexamic acid, or endometrial or cervical malignancies. Patients receiving gonadotrophin-releasing hormone $(\mathrm{GnRH})$ analogues or anticoagulant therapy were also excluded.

A total of 60 patients scheduled for hysteroscopic myomectomy that fulfilled the inclusion and exclusion criteria were enrolled in the study after explaining the procedure and taking an informed written consent to participate.

The patients were randomized into 2 groups using a computer-generated randomisation list. Randomized allocation was kept in opaque sealed envelopes with enrollment number written on the envelopes. Each envelope had a card on which the study group was written. The seal was broken only after enrolment into the study.

All patients had preoperative evaluation by gynecologists and anesthetists, with history taking, pelvic examination, transvaginal ultrasonography. Prior diagnostic hysteroscopy was done for all patients. Transvaginal ultrasonography was done to determine the number, size and location of the myomas. Also, evaluation of the thickness of the myometrium between intramural portion of the submucous uterine fibroid and the serosa of the uterine wall was done to prevent uterine perforation during the hysteroscopic resection. An ideal thickness was estimated to be between 5 and $10 \mathrm{~mm}$, according to Wong and Brun. ${ }^{11}$

Diagnostic hysteroscopy was performed for evaluation of the number, location and grade of the submucous myomas, using the Wamsteker grading system. Also, assessment of endocervical canal and uterine cavity and the position of the tubal ostia was done to aid orientation. $^{5,11}$

Preoperative full blood count, serum creatinine, fasting blood glucose, liver enzymes, coagulation profile (PT, PTT, INR), and viral markers were done for all the patients. The mode of anesthesia was determined by anesthetists not involved in the study.

After enrollment, patients were randomized into one of these 2 groups:

\section{Group A (Tranexamic acid group)}

Patient received $10 \mathrm{mg} / \mathrm{kg}$ of tranexamic acid, (Kapron ${ }^{\circledR}$ amp $5 \mathrm{ml}$, 100mg/ml; manufactured by Amoun Pharmaceutical Co., Cairo, Egypt). It was given slowly intravenously over 10 minutes immediately before the operation after induction of anesthesia.

\section{Group B (Oxytocin group)}

Patients received an infusion of 10 IU of oxytocin (Syntocinon ${ }^{\circledR}$ amp $10 \mathrm{IU} / 1 \mathrm{ml}$; manufactured by Novartis 
Pharma Co., Cairo, Egypt), added to $100 \mathrm{ml}$ normal saline solution running at a rate of $400 \mathrm{mIU} / \mathrm{min}$ (4 $\mathrm{ml} / \mathrm{min}$ ) throughout the procedure.

The procedure was done using the available standard setup of the ECDU, which is a 26-Fr monopolar resectoscope (Karl Storz, Tuttlingen, Germany) equipped with a loop electrode using a standard slicing technique. The distending solution used was $1.5 \%$ glycine.

Postoperative full blood count was done for all patients 24 hours after the procedures.

Preoperative and postoperative hemoglobin and hematocrit, the need for blood transfusion, the duration of operation, any operative complications and medication adverse effects were recorded.

\section{Statistical analysis}

The collected data were revised, coded, tabulated and processed using Statistical Package for Social Science (SPSS 20). Data were presented and suitable analysis was done according to the type of data obtained for each parameter.

The Mean and Standard deviation $( \pm$ SD) were used for parametric numerical data, while Median and Interquartile range (IQR) were used for non-parametric numerical data. The T-Test was used to assess the statistical significance of the difference between the two study group means. The Mann Whitney Test (U test) was used to assess the statistical significance of the difference of a non-parametric variable between two study groups.

The Chi-Squared test was used to examine the relationship between two qualitative variables, and the Fisher's exact test was used to examine the relationship between two qualitative variables when the expected count is less than 5 in more than $20 \%$ of cells.

\section{RESULTS}

The mean age of the study population was 38.9 years $( \pm 4.76)$. Twenty-two $(36.7 \%)$ of the patients were nulligravidas, while $38(63.3 \%)$ were multigravidas. Twenty-six (43.3\%) of the ladies nulliparous, $24(40 \%)$ delivered 1-3 times, while $10(16.7 \%)$ delivered more than 3 times. Fifty-one $(85 \%)$ of the women never had a miscarriage, 6 (10\%) had between 1-3 miscarriages, while 3 of them (5\%) had more than 3 miscarriages. The 2 study groups were demographically matched, as there was no statistically significant difference between them regarding the aforementioned criteria, as shown in Table 1.

Additionally, the sizes and grades of the myomas were comparable in both groups, with no statistically significant difference (Table 2). All included patients had a single submucous myoma. Eight patients $(26.7 \%)$ in Group I, and 4 patients (13.3\%) in Group II had a Grade 0 myoma (totally intra-cavitary). Twenty-two $(73.3 \%)$ of patients in Group I, and $26(86.7 \%)$ in Group II, had a Grade I myoma (more than $50 \%$ intra-cavitary). The median myoma size in Group I was $3 \mathrm{~cm}$, while that in Group II was $2 \mathrm{~cm}$. This was a statistically nonsignificant difference.

As shown Table 3, the mean difference between the preoperative and postoperative hemoglobin $(\mathrm{Hb})$ levels for patients in the oxytocin group was $-1.27( \pm 0.84)$, while in the tranexamic acid group, it was $-1.14( \pm 0.83)$ with no significant difference $(\mathrm{p}=0.558)$. By percentage, the hemoglobin level was reduced by $11.06 \%$ with SD \pm 6.46 in the oxytocin group, and by $10.21 \%$ (SD \pm 7.29 ) in tranexamic acid group with no significant difference $(p=0.636)$.

Table 1: Demographic data of the two study groups.

\begin{tabular}{|c|c|c|c|c|c|}
\hline Parameter & Tranexamic Acid (N=30) & Oxytocin (N=30) & Total $(\mathbf{N}=60)$ & Test & p-value \\
\hline Age (years) & $38.67 \pm 5.69$ & $39.13 \pm 3.82$ & $38.90 \pm 4.76$ & $\mathrm{t}=0.711$ & 0.291 \\
\hline \multicolumn{6}{|l|}{ Parity } \\
\hline NG & $12(40.0 \%)$ & $10(33.3 \%)$ & $22(36.7 \%)$ & \multirow{2}{*}{$C=0.592$} & \multirow{2}{*}{0.481} \\
\hline MP & $18(60.0 \%)$ & $20(66.7 \%)$ & $38(63.3 \%)$ & & \\
\hline \multicolumn{6}{|c|}{ Number of deliveries } \\
\hline $\mathrm{P} 0+$ & $16(53.3 \%)$ & $10(33.3 \%)$ & $26(43.3 \%)$ & \multirow{4}{*}{$\mathrm{F}=0.154$} & \multirow{4}{*}{0.244} \\
\hline $\mathrm{P} 1-3$ & $8(26.7 \%)$ & $16(53.3 \%)$ & $24(40.0 \%)$ & & \\
\hline $\mathrm{P}>3$ & $6(20 \%)$ & $4(13.3 \%)$ & $10(16.7 \%)$ & & \\
\hline Median (IQR) & $0.00(0-3)$ & $2.00(0-3)$ & $0.00(0-2)$ & & \\
\hline \multicolumn{6}{|c|}{ Number of miscarriages } \\
\hline 0 & $25(83.3 \%)$ & $26(86.7 \%)$ & $51(85 \%)$ & \multirow{4}{*}{$\mathrm{F}=0.005$} & \multirow{4}{*}{0.874} \\
\hline $1-3$ & $4(13.3 \%)$ & $2(6.7 \%)$ & $6(10 \%)$ & & \\
\hline$>3$ & $1(3.3 \%)$ & $2(6.7 \%)$ & $3(5 \%)$ & & \\
\hline Median (IQR) & $0.00(0-1)$ & $0.00(0-0)$ & $0.00(0-1)$ & & \\
\hline
\end{tabular}

(T) T-test of significance. (C) Chi-Square test of significance. (IQR) Interquartile range. (F) Fisher's Exact test of significance 
Table 2: Myoma characteristics in the study groups.

\begin{tabular}{|c|c|c|c|c|c|}
\hline Parameter & Tranexamic acid $(\mathbf{N}=\mathbf{3 0})$ & Oxytocin $(\mathbf{N}=30)$ & Total $(\mathrm{N}=60)$ & Test & p-value \\
\hline \multicolumn{6}{|c|}{ Grade of myoma } \\
\hline Grade 0 & $8(26.7 \%)$ & $4(13.3 \%)$ & $12(20 \%)$ & \multirow{2}{*}{$\mathrm{C}=0.197$} & \multirow{2}{*}{0.371} \\
\hline Grade 1 & $22(73.3 \%)$ & $26(86.7 \%)$ & $48(80 \%)$ & & \\
\hline \multicolumn{6}{|c|}{ Size of myoma $(\mathrm{cm})$} \\
\hline Median (IQR) & $3.00(2-4)$ & $2.00(2-4)$ & $2(2-40)$ & $\mathrm{M}=0.12$ & 0.441 \\
\hline
\end{tabular}

(C) Chi-Square test of significance. (IQR) Interquartile range. (M) Mann-Whitney test of significance

Table 3: The hemoglobin and hematocrit differences (absolute value and percentage) in both study groups.

\begin{tabular}{|llll|}
\hline Parameter & Tranexamic acid $(\mathrm{N}=30)$ & Oxytocin $(\mathrm{N}=30)$ & $\mathrm{t}$ test $(\mathrm{p}$-value) \\
\hline Hb difference & $-1.14 \pm 0.83$ & $-1.27 \pm 0.84$ & 0.558 \\
\hline Htc difference & $-4.54 \pm 2.80$ & $-4.29 \pm 2.86$ & 0.730 \\
\hline Hb \% reduction & $10.21 \pm 7.29$ & $11.06 \pm 6.46$ & 0.636 \\
\hline Htc \% reduction & $12.91 \pm 7.58$ & $11.83 \pm 7.34$ & 0.577 \\
\hline
\end{tabular}

Table 4: Comparison between the 2 study groups as to the operative time and blood component transfusion.

\begin{tabular}{|llll|}
\hline Parameter & Tranexamic acid $(\mathbf{N}=30)$ & Oxytocin $(\mathbf{N}=30)$ & p-value \\
\hline Time of operation $(\mathbf{m i n})$ & & $25(15-25)$ & $0.63(\mathrm{M})$ \\
\hline Median (IQR) & $20(15-25)$ & 0 & --- \\
\hline $\begin{array}{l}\text { Blood component } \\
\text { transfusion }\end{array}$ & 0 & 0 & - \\
\hline
\end{tabular}

(M) Mann-Whitney test of significance. (IQR) Interquartile range

In oxytocin group, the mean difference between preoperative and postoperative hematocrit (Hct) levels was $-4.29( \pm 2.86)$, while in the tranexamic acid group, it was $-4.54( \pm 2.80)$ with no significant difference $(p=$ 0.730 ). Hematocrit levels were reduced by $11.83 \%$ (SD \pm 7.34 ) in the oxytocin group, and by $12.91 \%(\mathrm{SD} \pm 7.58)$ in tranexamic acid group with no significant difference $(\mathrm{p}=0.577)$.

No patients needed blood component transfusion in either group. There was no significant difference between the two groups regarding duration of operation $(p=0.63)$ (Table 4). Uterine perforation was the only operative complication that occurred during the study. This occurred in one case in each group. Both cases were due to false passage of the Heggar's dilators used to dilate the cervix in order to pass the resectoscope. Both cases were managed conservatively, with no further complications, and the procedures postponed.

Nausea was the only reported medications adverse effect recorded during the study. It occurred in 3 cases of oxytocin group and in 2 cases of tranexamic acid group with no statistical difference between 2 groups $(\mathrm{p}=0.643)$. The patients reported none of the other investigated adverse effects (headaches, syncope, or nasal congestion), and there were no arrhythmias observed by the anaesthestists. No cases were reported to show thrombotic events within one week postoperatively.

\section{DISCUSSION}

This was a randomized controlled trial, comparing the safety and efficacy of oxytocin versus tranexamic acid for reducing the blood loss during hysteroscopic myomectomy. The study recruited 60 patients, divided into 2 equal groups, over one year.

The two study groups were comparable regarding their demographic criteria (age, parity, number of deliveries, and number of abortions), as well as the myoma characteristics (size and grade). There was no significant difference between the two groups regarding duration of operation, and none of the patient needed blood component transfusion.

There was no significant difference between the 2 study groups regarding the mean difference between preoperative and postoperative hemoglobin (absolute value and percentage; $\mathrm{P}=0.558, \mathrm{P}=0.636$ respectively). Additionally, there was no significant difference between the 2 study groups regarding the mean difference between preoperative and postoperative hematocrit (absolute value and percentage; $\mathrm{P}=0.730, \mathrm{P}=0.577$ respectively).

To the best of our knowledge, only two studies have compared an antifibrinolytic drug versus oxytocin as regard their effect in reducing blood loss in operative hysteroscopy. 
Mousa and colleagues recruited 50 women scheduled for hysteroscopic myomectomy, and randomly assigned them to receive either tranexamic acid $(15 \mathrm{mg} / \mathrm{kg})$, or oxytocin (at $400 \mathrm{mU} / \mathrm{min}$ ). ${ }^{12}$ Similar to our study, they reported no significant difference between the two groups regarding operative time or the need for blood transfusion. However, they reported a significant decrease of the postoperative hemoglobin and hematocrit values in the tranexamic acid group. They have concluded that this was a result of the uterotonic effect of oxytocin which might be more effective in reducing surgical blood loss than the antifibrinolytic effect of tranexamic acid.

The second study was conducted by Sayyahmelli et al, who aimed to compare the efficacy and safety of intrauterine epsilon-aminocaproic acid (EACA) and intravenous oxytocin in the management of intractable uterine bleeding during operative hysteroscopy. ${ }^{13}$

As in our study, there was no statistical significant difference in serum hematocrit change in oxytocin group versus the EACA group, neither was there a significant difference in hemoglobin changes between the 2 groups. However, and unlike the results in our study, they found that the mean operative time was significantly shorter in patients who received intrauterine instillation of EACA, and hence, recommended the use of EACA in managing intractable uterine bleeding in candidates of hysteroscopy.

In the previous reported studies, no intraoperative or postoperative complications were reported in either group. ${ }^{12,13}$ Differently, uterine perforation was the only intraoperative complication occurred (no cases of cervical tears or fluid overload). This complication occurred in one case in each group, and was due to false passage of the cervical dilators prior to the introduction of the resectoscope. The 2 cases were kept under observation and given parenteral broad spectrum antibiotics, before being discharging home after assurance of absence of further complications. This might be attributed to the relatively larger size of the used resectoscope in our study (26 Fr), that needed vigorous cervical dilatation.

The only medications adverse effect encountered was postoperative nausea, with no significant difference between the 2 study groups $(\mathrm{P}=0.643)$. No thromboembolic complications reported 1 week after operation in both groups.

This current study comes forward as one of a very few comparing the safety and efficacy of oxytocin versus tranexamic acid in reducing perioperative blood loss during hysteroscopic myomectomy. This study, however, had some limitations, including that the number of patients was not enough to detect uncommon medications side effects, and that it included mostly small sized myomata and all its patients had only a single submucous myoma.

\section{CONCLUSION}

The results of this study indicated that tranexamic acid and oxytocin are equally effective in reducing blood loss and transfusion requirements in hysteroscopic myomectomy with good safety profiles. Both of them can be used according to the availability, and surgical team preferences.

\section{ACKNOWLEDGMENTS}

Authors would like to thank the generous help and support of all ECDU personnel of the Ain Shams University Maternity Hospital.

Funding: No funding sources

Conflict of interest: None declared

Ethical approval: The study was approved by the Institutional Ethics Committee

\section{REFERENCES}

1. Falcone T, Parker WH. Surgical management of leiomyomas for fertility or uterine preservation. Obstetrics Gynecol. 2013;121(4):856-68.

2. Baird DD, Dunson DB, Hill MC, Cousins D, Schectman JM. High cumulative incidence of uterine leiomyoma in black and white women: ultrasound evidence. Am J Obst Gynecol. 2003;188(1):100-7.

3. Frederick S, Frederick J, Fletcher H, Reid M, Hardie M, Gardner W. A trial comparing the use of rectal misoprostol plus perivascular vasopressin with perivascular vasopressin alone to decrease myometrial bleeding at the time of abdominal myomectomy. Fertility Sterility. 2013;100(4):10449.

4. Raga F, Sanz-Cortes M, Bonilla F, Casañ EM, Bonilla-Musoles F. Reducing blood loss at myomectomy with use of a gelatin-thrombin matrix hemostatic sealant. Fertility Sterility. 2009;92(1):356-60.

5. Saridogan E. Surgical treatment of fibroids in heavy menstrual bleeding. Women's Health. 2016;12(1):53-62.

6. Mazzon I, Bettocchi S, Fascilla F, Palma F, Zizolfi B. Resectoscopic myomectomy. Minerva Ginecol. 2016;68(3):334-44.

7. Kongnyuy EJ, Wiysonge CS. Interventions to reduce haemorrhage during myomectomy for fibroids. Cochrane Database Systematic Reviews. 2014. Available at https://www.cochranelibrary.com/cdsr/ doi/10.1002/14651858.CD005355.pub5/media/CDS R/CD005355/CD005355.pdf.

8. Murphy DJ, MacGregor H, Munishankar B, McLeod G. A randomised controlled trial of oxytocin 5IU and placebo infusion versus oxytocin 5IU and 30IU infusion for the control of blood loss at elective caesarean section-pilot study. ISRCTN 40302163. European Journal Obst Gynecol Repro Biol. 2009;142(1):30-3. 
9. McCormack PL. Tranexamic acid. Drugs. 2012;72(5):585-617.

10. Ido K, Neo M, Asada $\mathrm{Y}$, Kondo K, Morita T, Sakamoto $\mathrm{T}$, et al. Reduction of blood loss using tranexamic acid in total knee and hip arthroplasties. Arch Ortho Trauma Surg. 2000;120(9):518-20.

11. Wong L, Brun JL. Myomectomy: technique and current indications. Minerva Ginecol. 2014;66(1):3547.

12. Mousa SA, Yassen AM, Alhadary HS, Sadek EE, Abdel-Hady ES. Hematological profile and transfusion requirement during hysteroscopic myomectomy: a comparative study between oxytocin and tranexamic acid infusion. Egyptian $\mathrm{J}$ Anaesth. 2012;28(2):125-32.
13. Sayyahmelli M, Moosazadeh R, Mostafa GP, Nazari F. Comparison of the intraoperative outcomes of intrauterine instillation of epsilon-aminocaproic acid and intravenous oxytocin in hysteroscopy surgeries. Int J Women's Health Rep Sci. 2016;4(2):68-72.

Cite this article as: Mohamed SE, El Helaly AM, Salama MH. Effect of oxytocin infusion versus tranexamic acid on reducing blood loss during hysteroscopic myomectomy: a randomized controlled trial. Int J Reprod Contracept Obstet Gynecol 2019;8:2586-91. 